\title{
Qual Escore de Risco Melhor Avalia os Objetivos Clínicos para meu Paciente com Miocardiopatia Hipertrófica?
}

\section{Which Risk Score Best Assesses Clinical Objectives in Patients with Hypertrophic Cardiomyopathy?}

\author{
Eduardo Alberto de Castro Roque ${ }^{10}$ \\ Hospital Metropolitano, ${ }^{1}$ Serra, ES - Brasil \\ Minieditorial referente ao artigo: Discordância entre Diretrizes Internacionais sobre Critérios de Prevenção Primária de Morte Súbita na \\ Cardiomiopatia Hipertrófica
}

A cardiomiopatia hipertrófica $(\mathrm{CMH})$ é um distúrbio genético caracterizada pela hipertrofia inexplicada do ventrículo esquerdo, comumente assimétrico com maiores espessamentos do septo interventricular basal. A obstrução da via de saída do ventrículo esquerdo está presente em repouso em cerca de um terço dos pacientes e pode ser provocada em outro terço. As características histológicas da $\mathrm{CMH}$ incluem hipertrofia e desordem dos miócitos, além de fibrose intersticial. A hipertrofia também é frequentemente associada à disfunção diastólica do ventrículo esquerdo.

$\mathrm{O}$ primeiro caso de $\mathrm{CMH}$ foi descrito por Henri Liouville em 1869 no Gazette Medecine Paris. Em 1907, o Dr. A. Schmincke, patologista alemão, descreveu dois corações com hipertrofia ventricular esquerda; ambos vieram de mulheres na casa dos cinquenta anos. Levy e von Glahn, em 1944, da Universidade da Colômbia, em Nova York, publicaram uma série de casos que se assemelham a CMH. Em 1949, William Evans, um cardiologista de Londres, descreveu a ocorrência familiar de hipertrofia cardíaca em uma série de pacientes semelhantes aos descritos no artigo de Levy e von Glahn. Dr. Eugene Braunwald e Dr. Andrew Glenn Morrow publicaram uma série de trabalhos onde detalharam aspectos clínicos e hemodinâmicos desta doença permitindo estabelecer objetivos terapêuticos. ${ }^{1-5}$

Na maioria dos pacientes a $\mathrm{CMH}$ tem um curso relativamente benigno. Porém a CMH também é uma causa importante de morte súbita (MS), particularmente em adolescentes e adultos jovens, com risco de 0,5 a $2 \%$ ao ano, sendo a causa mais frequente de MS em adolescentes..$^{6,7}$ A prevenção de eventos de MS através de implante de um dispositivo de implantado parece óbvia, uma vez que as opções de tratamento clínico para prevenção de arritmia grave fatal não podem ser oferecida pelo tratamento farmacológico de forma confiável. Como uma terapia de elevado custo e não isenta de eventos adversos (infecção e choque inapropriado) tornou-se mandatório

\section{Palavras-chave}

Cardiomiopatia Hipertrófica Familiar; Programa de Vigilância de Riscos; Estenose Subaórtica Fixa; Desfibriladores Implantáveis; Hipertrofia Ventricular Esquerda.

Correspondência: Eduardo Alberto de Castro Roque •

Avenida Abido Saad, 2767. CEP 29175-585, Das Laranjeiras, Serra

ES - Brasil

E-mail: edcastro77@gmail.com, eduardo.roque@cardiol.br

DOI: https://doi.org/10.36660/abc.20200543 estabelecer quais grupos teriam benefício com a indicação de terapia com desfibrilador implantado.

Entre os escores de avaliação de risco de MS para definir os pacientes de maior benefício, ganharam destaques a calculadora de risco da AHA publicada em $2011^{8}$ e a calculadora de risco para avaliação de MS pela Sociedade Europeia de Cardiologia em 2015. ${ }^{9}$ Com metodologias diferentes ambas se propunham auxiliar o clínico a identificar um grupo de pacientes de maior benefício.

Um classificador ideal deveria ser simples, com poucos critérios, cada critério sendo de fácil interpretação, reproduzível com base na prática existente, altamente sensível, de alto valor preditivo negativo, capaz de reduzir riscos com menor custo possível. Ainda que atenda a todos os quesitos acima, temos que levar em consideração que os modelos prognósticos são desenvolvidos para serem aplicados em novos pacientes, que podem vir de diferentes centros, de diferentes raças, hábitos, morbidades distintas e com os mais diferentes microbiomas. Portanto, novos pacientes são comumente referidos como diferentes, mas semelhantes aos pacientes usados para desenvolver os modelos. Quando uma nova população de pacientes pode ser considerada semelhante (suficiente) à população em desenvolvimento para justificar a validação e, eventualmente, a aplicação de um modelo? A resposta a esta pergunta é totalmente dependente de registros médicos que possam revalidar ferramentas que usamos em nossa prática. ${ }^{10}$

O trabalho em questão ${ }^{11}$ vai além e avalia de forma prospectiva uma coorte de pacientes aplicando as ferramentas mais usadas na atualidade para validar qual delas seria de maior acurácia em nossa população, assim como identifica pontos fortes e fragilidades de nossa capacidade de avaliar e predizer eventos futuros. A importância do trabalho vai além do tema, uma vez que demonstra a necessidade de obtermos registros, permitindo que a comunidade cientifica seja capaz de revalidar os mais distintos escores clínicos em nossa prática. Com enorme impacto em eficácia (ao reduzir ocorrência de mortes súbitas) assim como de eficiência (ao permitir que possamos alocar recursos para contemplar os pacientes com maior chance de benefício). 


\section{Minieditorial}

\section{Referências}

1. Braunwald E (2012). "Hypertrophic cardiomyopathy: The first century 1869-1969". Glob Cardiol Sci Pract. 2012 (1):5

2. Evans W. "Familial cardiomegaly". Br Heart J.1949; 11(1):68-82.

3. Pare JA, Fraser RG, Pirozynski WJ,Shanks JA, Stubington D. "Hereditary cardiovascular dysplasia. A form of familial cardiomyopathy". Am J Med. 31: 37-62..

4. Teare D. "Asymmetrical hypertrophy of the heart in young adults". Br Heart J. 1958;20(1):1-8.

5. Brock R. "Functional obstruction of the left ventricle (acquired aortic subvalvar stenosis)". Guys Hosp Rep.1959;108:126-43.

6. Maron BJ (2003). "Sudden death in young athletes". N Engl J Med. 349(11):1064-75.

7. Shiozaki AA, Senra T, Arteaga E, Pita CG, Martinelli Filho M, Avila LF, et al. Myocardial fibrosis in patients with hypertrophic cardiomyopathy and high risk for sudden death. Arq Bras Cardiol.2010;94(4):535-40.
8. Gersh BJ, Maron BJ, Bonow RO, Dearani JA, Fifer MA, Link MS, et al. American College of Cardiology Foundation/American Heart Association task force on practice guidelines; American Association for Thoracic Surgery; American Society of Echocardiography; American Society of Nuclear Cardiology; Heart Failure Society of America; Heart Rhythm Society; Society for Cardiovascular Angiography and Interventions; Society of Thoracic Surgeons. 2011 ACCF/AHA guideline for the diagnosis and treatment of hypertrophic cardiomyopathy executive summary: a report of the American College of Cardiology Foundation/American Heart Association task force on practice guidelines. Circulation.2011;124(24):2761-96.

9. O'Mahony C, Jichi F, Pavlou M, Monserrat L, Anastasakis A, Rapezzi C, et al Hypertrophic cardiomyopathy outcomes investigators. A novel clinical risk prediction model for sudden cardiac death in hypertrophic cardiomyopathy (HCM risk-SCD). Eur HeartJ.2014;35(30):2010-20.

10. Moonsk, Altman DG, Vergouwe Y, Royston P. Prognosis and prognostic research: application and impact of prognostic models in clinical practice. BMJ. 2009 Jun 04;338:b606 doi: 10.1136/bmj.b606

11. Mattos BP, Scolari FL, Garbin HI . Discrepancy between International Guidelines on the Criteria for Primary Prevention of Sudden Cardiac Death in Hypertrophic Cardiomyopathy. Arq Bras Cardiol. 2020; 115(2):197-204). 